\title{
THE REAL COHOMOLOGY RING OF A SPHERE BUNDLE OVER A DIFFERENTIABLE MANIFOLD
}

\author{
BY
}

D. G. MALM

1. Introduction. The main result of this paper is already known; it follows from a theorem of A. Borel [2, paragraph 24] and has also been obtained by G. Hirsch [3]. However, it is believed that the proofs and descriptions of results given here are much simpler than in either of the above papers. The method used here is that of a previous paper [4] of the author; this paper could serve as an introduction to it.

We are concerned with the cohomology ring with real coefficients of a fiber space whose fiber is a $(k-1)$-sphere, for $k$ even. When given a cohomology theory whose cochains are anti-commutative, a description is given of the cohomology ring of the total space, in terms of the characteristic class $W_{k}$ of the sphere space, which is practical for computations in many cases. In $\$ 5$ these results are used to obtain, in a simplified form, the results of G. Hirsch [3] in terms of his "strict" triple products.

If the fibering sphere is of even dimension, then using real coefficients, $W_{k}=0$, and the cohomology ring is described in the paper [5] of W. S. Massey.

The author wishes to thank W. S. Massey for pointing out the simplification which occurs when given an anti-commutative ring of cochains, and for help in the preparation of this paper.

2. We suppose given a fiber space $\left(E, p, B, S^{k-1}\right)$ with fiber a $(k-1)$ sphere, $k$ even. By "fiber space" is meant a "locally trivial fiber space": For each $x \in B$, there is a neighborhood $V$ of $x$ and a homeomorphism $\phi$ mapping $V \times S^{k-1}$ onto $p^{-1}(V)$ such that $p \phi(y, z)=y$ for $y \in V$ and $z \in S^{k-1}$. We will assume that the sphere space is orientable in the following sense: If $S_{x}^{k-1}$ denotes the fiber over $x \in B$, then the local system of groups defined by $H^{k-1}\left(S_{x}^{k-1}\right)$, for $x \in B$, is a simple system. We also assume that the base space $B$ is compact, and that we are given a cohomology theory with real coefficients, whose cochains are anti-commutative. An example of this would be if $B$ were a differentiable manifold, the sphere space had a differentiable structure, and the ring of cochains were the exterior differential forms. However, it is possible to have a ring of anti-commutative cochains under less restrictive assumptions.

We recall a result of Thom [7]: If $A$ is the mapping cylinder of $p: E \rightarrow B$, the Gysin sequence of $\left(E, p, B, S^{k-1}\right)$ is isomorphic to the cohomology sequence of the pair $(A, E)$. In fact, there is an element $\mathfrak{U} \in H^{k}(A, E)$ such that

Received by the editors April 17, 1961. 
the homomorphism $\theta: H^{q-k}(A) \rightarrow H^{q}(A, E)$ defined by $\theta(x)=x \mathcal{u}$ (the cup product) is an isomorphism onto. The natural projection $p_{0}: A \rightarrow B$ of the mapping cylinder onto the base space induces an isomorphism $p_{0}{ }^{*}: H^{q}(B)$ $\rightarrow H^{q}(A)$. We then have the commutative diagram

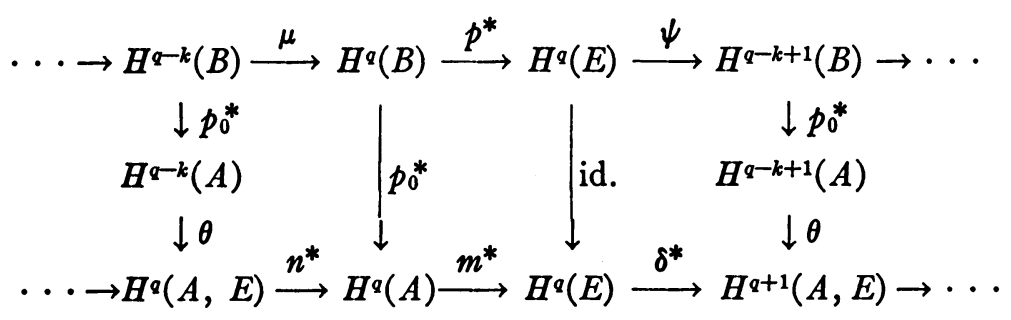

Figure 1

In this diagram, the top line is the Gysin sequence and the bottom line is the cohomology sequence of the pair $(A, E)$. According to the results of Thom, $n^{*}(\mathcal{u})=p_{0}^{*}\left(W_{k}\right)$.

We will regard $C^{*}(A, E)$ as a subgroup of $C^{*}(A)$. It is actually an ideal in $C^{*}(A)$ and $C^{*}(E) \approx C^{*}(A) / C^{*}(A, E)$. Making this identification, $n^{*}$ is induced by the inclusion $C^{*}(A, E) \subset C^{*}(A)$, and $m^{*}$ is induced by the natural map $m^{*}: C^{*}(A) \rightarrow C^{*}(A) / C^{*}(A, E)$, where $m: E \rightarrow A$ is the inclusion.

3. Let $V$ be a representative cocycle for the characteristic class $W_{k} \in H^{k}(B)$. Construct the algebraic mapping cylinder $M$ of the map $x \rightarrow x V$ for $x \in C^{*}(B)$, that is, let

$$
\begin{aligned}
M^{p} & =C^{p}(B) \times C^{p-k+1}(B) \\
M & =\sum_{p} M^{p}
\end{aligned}
$$

and

$$
\delta(x, y)=(\delta x+y V,-\delta y) \quad \text { for }(x, y) \in M .
$$

It is easily seen that $(M, \delta)$ is a differential group, with addition defined componentwise.

We now introduce a multiplication in $M$, by the formula

$$
(x, y)(v, w)=\left(x v,(-1)^{p} x w+y v\right)
$$

for $(x, y) \in M^{p}$ and $(v, w) \in M^{q}$. The product has degree $p+q$, and is associative and anti-commutative. It is easily verified that $\delta[(x, y)(v, w)]$ $=[\delta(x, y)](v, w)+(-1)^{p}(x, y) \delta(v, w)$ for $(x, y) \in M^{p}$. Consequently a product is induced in the derived group $H^{*}(M)$.

Theorem I. Using this product, $H^{*}(M)$ is isomorphic to $H^{*}(E)$ as an algebra over the reals. 
To prove this theorem, we consider the diagram of exact sequences

$$
\begin{aligned}
0 & \rightarrow C^{p}(B) \stackrel{i}{\rightarrow} M^{p} \stackrel{j}{\rightarrow} C^{p-k+1}(B) \rightarrow 0 \\
\downarrow \delta & \delta \downarrow \\
0 & \rightarrow C^{p+1}(B) \stackrel{i}{\rightarrow} M^{p+1} \stackrel{\downarrow}{\rightarrow} C^{p-k+2}(B) \rightarrow 0
\end{aligned}
$$

where $i(x)=(x, 0)$ for $x \in C^{*}(B)$ and $j(x, y)=y$ for $(x, y) \in M$. The left square commutes and the right square anti-commutes. From this diagram we obtain the exact cohomology sequence of the algebraic mapping cylinder:

$$
\cdots \rightarrow B^{p-k}(B) \stackrel{\mu}{\rightarrow} H^{p}(B) \stackrel{i^{*}}{\rightarrow} B^{p}(M) \stackrel{j^{*}}{\rightarrow} H^{p-k+1}(B) \rightarrow \cdots
$$

Here, $\mu$ is induced by $x \rightarrow x V$, in other words $\mu$ is the same map as in the Gysin sequence. We have the diagram

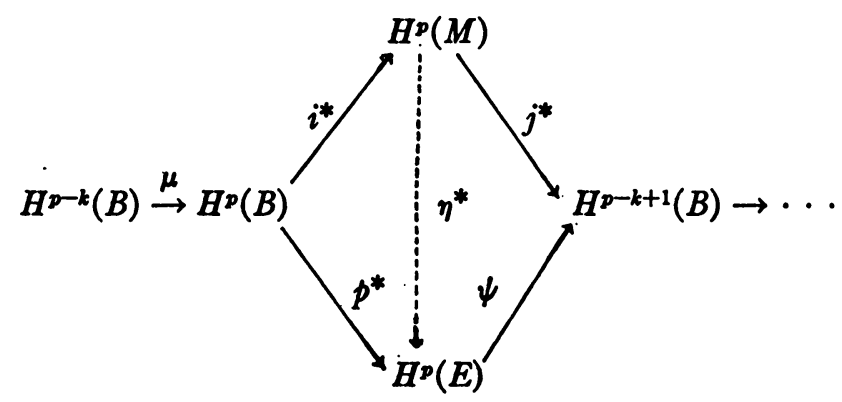

We will define a ring homomorphism $\eta^{*}: H^{p}(M) \rightarrow H^{p}(E)$ for which $\eta^{*} i^{*}$ $=p^{*}$ and $-j^{*}=\psi \eta^{*}$; it will then follow by the five-lemma that $\eta^{*}$ is an isomorphism onto.

To define $\eta^{*}$, let $V^{\prime} \in C^{k}(A, E)$ be a representative cocycle for Thom's class $u \in H^{k}(A, E)$. Then for some $\alpha \in C^{k-1}(A), p_{0}^{\ddagger}(V)=V^{\prime}+\delta \alpha$. Here, $p_{0}^{t}$ is the cochain homomorphism induced by $p_{0}: A \rightarrow B$. Define

$$
\eta: M^{p} \rightarrow C^{p}(A) / C^{p}(A, E)=C^{p}(E)
$$

by

$$
\eta(x, y)=m^{t}\left(p_{0}^{t}(x)+(-1)^{p+1}\left(p_{0}^{\prime} y\right) \alpha\right) \quad \text { for }(x, y) \in M^{p} .
$$

It is easily verified that $\delta \eta=\eta \delta$ and that $\eta$ is an additive homomorphism. Also, for $(x, y) \in M^{p}$ and $(u, v) \in M^{q}$, a straightforward computation shows that

$$
\eta\{(x, y)(u, v)\}-\eta(x, y) \eta(u, v)=m^{\dagger}(\Gamma)
$$

where 


$$
\Gamma=(-1)^{p+q+1} p_{0}^{t}(y u) \alpha+(-1)^{p}\left(p_{0}^{t} y\right) \alpha\left(p_{0}^{t} u\right)+(-1)^{p+q+1}\left(p_{0}^{\dagger} y\right) \alpha\left(p_{0}^{t} v\right) \alpha .
$$

$\Gamma=0$ since the cochains are anti-commutative, and $\alpha^{2}=0$; therefore the induced map $\eta^{*}: H^{*}(M) \rightarrow H^{*}(E)$ is a ring homomorphism.

To prove the commutativity relations, let square brackets denote cohomology classes in the appropriate cohomology groups. For $[x] \in H^{p}(B)$, $\eta^{*} i^{*}[x]=\eta^{*}[(x, 0)]=\left[m^{*} p_{0}^{*} x\right]=p^{*}[x]$, in view of the commutativity of Figure 1. For $[(x, y)] \in H^{p}(M)$,

$$
\begin{aligned}
\left(\psi \eta^{*}\right)[(x, y)] & =\psi\left[m^{+}\left(p_{0}^{\dagger} x+(-1)^{p+1}\left(p_{0}^{\dagger} y\right) \alpha\right)\right] \\
& =p_{0}^{*-1} \theta^{-1} \delta^{*}\left[m^{\dagger}\left(p_{0}^{\dagger} x+(-1)^{p+1}\left(p_{0}^{\dagger} y\right) \alpha\right)\right] \\
& =p_{0}^{*-1} \theta^{-1}\left[p_{0}^{\dagger}(\delta x)+\left(p_{0}^{\dagger} y\right)\left(p_{0}^{\dagger} V-V^{\prime}\right)\right] \\
& =p_{0}^{*-1} \theta^{-1}\left[-\left(p_{0}^{\dagger} y\right) V^{\prime}\right]=-[y]=-j^{*}[(x, y)] .
\end{aligned}
$$

We remark that the algebra $H^{*}(M)$ is independent of the choice $V \in W_{k}$, as this theorem shows.

4. In this section we prove two propositions which should be useful in actual computations. Suppose $C$ and $\bar{C}$ are graded, anti-commutative cochain rings, and $V$ and $\bar{V}$ are $C$ and $\bar{C} k$-cocycles, respectively. Let $f: C \rightarrow \bar{C}$ be an allowable homomorphism for which $f(V)=\bar{V}$. Let $M$ and $\bar{M}$ be the algebraic mapping cylinders of $x \rightarrow x V$ and $x \rightarrow x \bar{V}$ respectively.

Proposition A. There is an allowable homomorphism $\phi: M \rightarrow \bar{M}$, which preserves products, induced by $f$. The homomorphism $\phi^{*}: H^{*}(M) \rightarrow H^{*}(\bar{M})$ induced by $\phi$ commutes with the homomorphisms of the algebraic mapping cylinders. Furthermore if $f^{*}: H^{*}(C) \rightarrow H^{*}(\bar{C})$ is an isomorphism onto, then so is $\phi^{*}$.

To prove this, define $\phi$ by $\phi(x, y)=(f x, f y)$ for $(x, y) \in M$. All the computations are straightforward. The last assertion follows from the fivelemma.

Now let $M^{n}$ be a compact, connected, differentiable $n$-dimensional manifold, with $H^{1}\left(M^{n}\right)=0$. Let $C^{*}$ be the algebra of exterior differential forms, with differential operator $d$.

Proposition $\mathrm{B}\left({ }^{1}\right)$. It is possible to choose a finitely generated subring $A^{*}$ of $C^{*}$, for which the inclusion $i: A^{*} \rightarrow C^{*}$ induces an isomorphism onto $i^{*}: H^{*}\left(A^{*}\right)$ $\rightarrow H^{*}\left(C^{*}\right)$.

Proof. Let $1, a_{1}, \cdots, a_{k}$ be a minimal set of generators of $H^{*}\left(C^{*}\right)$, with representative cocycles $1^{\prime}, a_{1}^{\prime}, \cdots, a_{k}^{\prime}$. These generate $A \subset C^{*}$, and the inclusion $i: A \rightarrow C^{*}$ induces $i^{*}: H^{*}(A) \rightarrow H^{*}\left(C^{*}\right)$ which is onto. The kernel of $i^{*}$ is generated as an ideal by $b_{1}, \cdots, b_{l}$; choose $b_{1}^{\prime}, \cdots, b_{l}^{\prime} \in C^{*}$ for which $d b_{i}^{\prime}=b_{i}$. Let $A^{\prime}$ be generated by $1, a_{1}^{\prime}, \cdots, a_{k}^{\prime}, b_{1}^{\prime}, \cdots, b_{l}^{\prime}$; make this set of

(1) This was suggested by W. S. Massey. 
generators minimal. All of the $b_{i}$ have degree at least three, for they arise from cocycles $a_{i}^{\prime}, a_{j}^{\prime}$ for which $a_{i}^{\prime} a_{j}^{\prime}$ is a coboundary, and $a_{i}^{\prime} a_{j}^{\prime}$ has degree at least four. If $i^{* \prime}: H^{*}\left(A^{\prime}\right) \rightarrow H^{*}\left(C^{*}\right)$ is not $1-1$, repeat the process, adding $c_{i}^{\prime} \mathrm{s}$ which have degree at least four. The process must end, for $C^{p}=0$ for $p>n$.

Using these two propositions, one can compute the cohomology rings in many cases.

5. We now suppose that the base space $B$ is a differentiable manifold, with a Riemannian metric, and we will use the exterior differential forms as cochains on $B$. Under these conditions, there are canonical additive homomorphisms ( $Z$ and $B$ represent cocycles and coboundaries)

and

$$
\alpha: H^{p}(B) \rightarrow Z^{p}(B)
$$

$$
\beta: \mathbb{B}^{p}(B) \rightarrow C^{p-1}(B) .
$$

$\alpha$ assigns to each cohomology class a representative cocycle (a harmonic form) and $\beta$ assigns to each $p$-coboundary a $(p-1)$-cochain such that $\delta \beta$ =identity. (See [1] or [6]; an outline of the results is given in [3].)

We construct the algebraic mapping cylinder of $x \rightarrow x \alpha\left(W_{k}\right)$ for $x \in C^{*}(B)$. This gives the exact sequence

$$
0 \rightarrow \frac{H^{p}(B)}{W_{k} \cdot H^{p-k}(B)} \stackrel{i}{\rightarrow} H^{p}(M) \stackrel{j}{\rightarrow}(\operatorname{kernel} \mu)^{p-k+1} \rightarrow 0
$$

where $i$ and $j$ are induced by $i^{*}$ and $j^{*}$ in the obvious ways, and

$$
(\text { kernel } \mu)^{p-k+1}=(\text { kernel } \mu) \cap H^{p-k+1}(B) .
$$

Note that a multiplication

$$
\frac{H^{p}(B)}{W_{k} \cdot H^{p-k}(B)} \times \text { kernel } \mu \rightarrow \operatorname{kernel} \mu
$$

is defined. This sequence splits; we define $\theta$ : (kernel $\mu)^{p-k+1} \rightarrow H^{p}(M)$ by requiring $\theta(y)$ to be the cohomology class in $H^{*}(M)$ of $\left(-\beta\left(\alpha y \cdot \alpha W_{k}\right), \alpha y\right)$. It is easily verified that $\theta$ is additive and $j \theta=$ identity. Thus every $x \in H^{*}(M)$ may be written uniquely in the form $x=i\left(x_{1}\right)+\theta\left(x_{2}\right)$, where $x_{2}=j(x)$. The product of two elements may be computed as follows ( $p$ is the degree of $x_{1}$ ):

$\left(i\left(x_{1}\right)+\theta\left(x_{2}\right)\right)\left(i\left(y_{1}\right)+\theta\left(y_{2}\right)\right)=i\left(x_{1} y_{1}\right)+\theta\left(x_{2}\right) i\left(y_{1}\right)+i\left(x_{1}\right) \theta\left(y_{2}\right)+\theta\left(x_{2}\right) \theta\left(y_{2}\right)$.

Applying $j$ to both sides, we get

$$
\begin{aligned}
& j\left\{\left(i\left(x_{1}\right)+\theta\left(x_{2}\right)\right)\left(i\left(y_{1}\right)+\theta\left(y_{2}\right)\right)\right\} \\
& \quad=j\left(\theta\left(x_{2}\right) i\left(y_{1}\right)\right)+j\left(i\left(x_{1}\right) \theta\left(y_{2}\right)\right)+j\left(\theta\left(x_{2}\right) \theta\left(y_{2}\right)\right) \\
& \quad=x_{2} y_{1}+(-1)^{p} x_{1} y_{2}+j\left(\theta\left(x_{2}\right) \theta\left(y_{2}\right)\right),
\end{aligned}
$$

since $j\left(\theta\left(x_{2}\right) i\left(y_{1}\right)\right)=x_{2} y_{1}$ and $j\left(i\left(x_{1}\right) \theta\left(y_{2}\right)\right)=(-1)^{p} x_{1} y_{2}$. To prove this last rela- 
tion, let $x_{1}^{\prime} \in H^{p}(B)$ represent $x_{1}$; then $i\left(x_{1}\right)$ is represented by $\left(\alpha x_{1}^{\prime}, 0\right) \in M^{p}$. $\theta\left(y_{2}\right)$ is represented by $\left(-\beta\left[\left(\alpha y_{2}\right)\left(\alpha W_{k}\right)\right], \alpha y_{2}\right)$. Using the product formula in $M^{p}$, we obtain $j\left(i\left(x_{1}\right) \theta\left(y_{2}\right)\right)=(-1)^{p} x_{1} y_{2}$. The other formula is proved similarly.

From the above equation $\left({ }^{*}\right)$ we obtain

$$
\left(i\left(x_{1}\right)+\theta\left(x_{2}\right)\right)\left(i\left(y_{1}\right)+\theta\left(y_{2}\right)\right)=i\left(x_{1} y_{1}\right)+\theta\left(x_{2} y_{1}+(-1)^{p} x_{1} y_{2}+j\left(\theta x_{2} \cdot \theta y_{2}\right)\right) .
$$

We may compute $j\left(\theta x_{2} \cdot \theta y_{2}\right)$; it is the cohomology class of

$$
-\left(\alpha x_{2}\right) \beta\left(\alpha y_{2} \cdot \alpha W_{k}\right)+(-1)^{p+1} \beta\left(\alpha x_{2} \cdot \alpha W_{k}\right) \alpha\left(y_{2}\right) .
$$

However, this is just $(-1)^{p+1}\left\langle x_{2}, W_{k}, y_{2}\right\rangle$, the triple product in the strict sense of Hirsch [3]. We thus have the following theorem.

THEOREM II. For the base space B a differentiable manifold with Riemannian metric, we have

$$
H^{p}(E) \approx \frac{H^{p}(B)}{W_{k} \cdot H^{p-k}(B)} \oplus(\text { kernel } \mu)^{p-k+1}
$$

The multiplication is given in terms of the strict triple product by

$$
\left(x_{1}, x_{2}\right)\left(y_{1}, y_{2}\right)=\left(x_{1} y_{1}, x_{2} y_{1}+(-1)^{p} x_{1} y_{2}+(-1)^{p+1}\left\langle x_{2}, W_{k}, y_{2}\right\rangle\right) \text {. }
$$

Theorem II is simpler than the corresponding result of Hirsch [3]; Hirsch uses several cohomology operations. In the above formulation, $x_{1}$ and $y_{1}$ are elements of $H^{*}(B) / W_{k} \cdot H^{*}(B)$. The author believes that the extra operations that Hirsch uses only lead to different representative elements for $x_{1}$ and $y_{1}$, and thus give the same product.

\section{REFERENCES}

1. P. Bidal and G. deRham, Les formes differentielles harmoniques, Comment. Math. Helv. 19 (1946), 1-49.

2. A. Borel, Sur la cohomologie des espaces fibres principaux et des espaces homogènes de groupes de Lie compacts, Ann. of Math. 57 (1953), 115-207.

3. G. Hirsch, Sur certaines operations dans l'homologie des espaces de Riemann, Bull. Soc. Math. Belg. 9 (1957), 115-139.

4. D. G. Malm, Concerning the cohomology ring of a sphere bundle, Pacific J. Math. 9 (1959), 1191-1214.

5. W. S. Massey, On the cohomology ring of a sphere bundle, J. Math. Mech. 7 (1958), 265290.

6. G. deRham, Variêtés différentiables, Actualités Sci. Ind., no. 1222, Hermann, Paris, 1955.

7. R. Thom, Espaces fibrés en sphìres et carrés de Steenrod, Ann. Sci. Ecole Norm. Sup. 69 (1952), 109-182.

State University of New York,

Oyster Bay, Long Island, New York 